\title{
Life under another sun: from science-fiction to science
}

Michaël Gillon

Initiated in the XVIth century, the Copernican revolution toppled our Earth from its theological pedestal, revealing it not to be the center of everything but a planet among several others in orbit around one of the zillions of stars of our Universe. Already proposed by some philosophers at the dawn of this major paradigm shift, the existence of exoplanets, i.e. planets in orbit around other stars than our Sun, remained suspected but unconfirmed for centuries. It is only in the last decade of the XXth century that the first of these extrasolar worlds were found. Their seminal discoveries initiated the development of more and more ambitious projects that led eventually to the detection of thousands of exoplanets, including a few dozen potentially habitable ones, i.e. Earth-like exoplanets that could harbor large amounts of liquid water -and maybe life- on their surfaces. Upcoming astronomical facilities will soon be able to probe the atmospheric compositions of some of these extrasolar worlds, performing maybe in the process the historical detection of chemical signs of life light-years away. But while the existence of extraterrestrial life remains pure speculation for now, it has been a major theme of science-fiction for more than a century. By creating countless stories of encounters between humans and alien forms of life, sciencefiction authors have pursued in a sense the Copernican revolution, confronting us to the ideas that not only life could be widespread in the Universe, but also that our species may be far from the Cosmic pinnacle in matter of intelligence and technological development.

\section{On the uniqueness of our world}

Since the dawn of civilization, some humans have wondered about the possible existence of other worlds in the Cosmos. In Ancient Greece, this cosmic pluralism hypothesis was championed by philosophers like Anaximander (610 - 546 BC), Democritus (460 - 370 BC) and Epicurus (341 - $270 \mathrm{BC}$ ), who believed in an infinite universe hosting an infinite number of inhabited worlds. Nevertheless, other prominent thinkers; like Plato (428-348 BC) and Aristotle (384-342 BC), argued that the Earth could only be unique, but also the center of the Cosmos. Their geocentric model was developed further by the Hellenistic astronomer Claudius Ptolemy $(100-170 \mathrm{AD})$, and later incorporated into the Christian dogma to become a standard feature of western medieval cosmology. In this paradigm, planets were eternally unchanging (and thus lifeless) bodies, and stars were just spots of light literally attached to a gigantic celestial sphere revolving around the fixed Earth in one day. It prevailed in Europa for more than a millennium, until the so-called Copernican Revolution, a paradigm shift initiated in $1543 \mathrm{AD}$ by the publication of De revolutionibus orbium coelestium by the Polish astronomer Nicolaus Copernicus' (1473-1543 AD). In this book, Copernicus presented a new cosmological model placing the Sun and not the Earth at the center of the Universe. This work remained mostly ignored for nearly eighty years, until it obtained critical support from observations made by astronomers like Tycho Brahe (1546 - 1601 AD), Galileo Galilei (1564 - 1642 AD), and Johannes Kepler (1571 - 1630 AD). Despite the strong resistance of the Catholic Church, the Ptolemaic system started buckling under the gradual accumulation of evidence for a heliocentric solar system. The final blow was brought in 1687 with the publication by Sir Isaac 
Newton (1643 - 1727 AD) of his Principia, a book in which he demonstrated that the motions of all the bodies of the solar system, including the orbits of Earth and other planets around the Sun, could be explained by a few physical postulates, his famous laws of motions and universal gravitation that laid the foundation for modern physics.

With the rise of the new heliocentric paradigm, some thinkers argued that the stars could be very remote Sun-like objects likewise orbited by planets. The first of them was the Italian philosopher Giordano Bruno (1548 - 1600 AD), who ended tried for heresy and burned at the stake by the Roman Inquisition. Remarkably, Bruno raised the possibility that some of these putative planets orbiting other suns (or exoplanets, as we call them today) might harbor life of their own. More than one century later, the great Sir Isaac Newton himself brought support to the Sun-like nature of the stars, and soon the idea reached consensus among astronomers, even if it was not yet firmly demonstrated by observations. This demonstration had to wait for the nineteenth century and the advent of stellar spectroscopy, pioneered by Joseph von Fraunhofer (1787 - 1826 AD) and Angelo Secchi (1818 - 1878), and the first determination of the distance of stars, pioneered by Friedrich Bessel (1784 - 1846 AD).

The Copernican Revolution made cosmic pluralism a mainstream notion, supported by serious treatises but also diffused to the public by more fictional works, like the famous Conversations on the Plurality of World, published in 1686 by the French scholar Bernard le Bovier de Fontenelle. At this point, it should be noted that while the Ptolemaic view of the Universe adopted by the Western civilization restrained the creation of fictions depicting, e.g., contacts with creatures of extraterrestrial origins during the whole medieval period ( $5^{\text {th }}$ to $15^{\text {th }}$ century $A D)$, it was not the case in other parts of the world. For instance, the Tale of the Bamboo Cutter, written in Japan in the $10^{\text {th }}$ century $A D$, tells the story of an extraterrestrial princess stranded on Earth, and rescued by his people after some adventures on our planet. Similar examples abound in many cultures, some dating back to Antiquity, like $A$ true Story written in the second century AD by Lucian of Samosata. Nevertheless, these ancient works were purely fictional, inspired by mythological or philosophical considerations, unlike the Conversations of Fontenelle and other contemporary works by Godwin, de Bergerac and more scholars, including the great Johannes Kepler himself with his book Somnium (1634 AD). These precursors planted the seed of science fiction, a new genre of fictional literature inspired by scientific theories and results, or dealing with their possible consequences.

\section{From planets to exoplanets}

The first astronomer to consider detecting exoplanets was Christian Huygens (1629 - 1695 $A D)$, who deemed such a detection impossible given the technology available at that time (Huygens 1698). Indeed, despite some attempts to detect exoplanets dating back to the nineteenth century, the first firm exoplanet detection had to wait for the last decade of the nineteenth century, as discussed below. Nevertheless, while exoplanets remained out of reach during the whole eighteenth and nineteenth centuries, instrumental astronomy developed then enough to make possible first detailed studies of the known other planets of our solar system (Mercury, Venus, Mars, Jupiter, Saturn), and the detection of two others, Uranus (in 1781) and Neptune (in 1846). It is thus no surprise that first speculations and fictions about the existence of extraterrestrial life focused mainly on our own planetary system. One of its planets in particular could capture the imagination of scientists and writers: Mars. 
Mars is the fourth planet from the Sun (Earth being the third), and the second-smallest planet in the solar system after Mercury. Its radius and mass are, respectively, twice and ten times smaller than Earth's. It can be seen easily from Earth with the naked eye, its distance at closest approach being of "only" 50 million kilometers. Its icy polar caps were discovered in the midseventeenth century, and proved to grown and shrink seasonally in the late eighteenth century. By the mid-nineteenth century, the planet was known to have several similarities to Earth, notably nearly exactly the same length of the day, and a similarly tilted rotation axis making it experience seasons just as Earth does. Speculation about life on Mars boomed in the late nineteenth century with the observation of apparent channels on its surface, first described in 1877 by the Italian astronomer Giovanni Schiaparelli (1835 - 1910 AD) and (wrongly) confirmed afterwards by other astronomers. A wealthy Bostonian businessman Percival Lowell (1855 - 1916 AD) championed the idea that these channels were in fact irrigation canals by an advanced civilization to face the dearth of water upon most of the planet's surface. The scientific community was skeptical about this idea, but it excited the public and inspired several influential classics of science fiction like H. G. Wells' The War of the Worlds (1898) that tells the story of an invasion of the Earth by Martians fleeing from their desiccated world. Many more novels on this theme appeared in the following decades, making enter the concept of "little green men from Mars" into popular culture.

Alas (or not), improved astronomical observations of Mars performed during the early twentieth century revealed its channels to be an optical illusion, and the planet's atmosphere to be extremely scarce and devoid of oxygen or water. In the next decades, astronomical observations made more and more unlikely any large surface biosphere on Mars, and the first space probes to reach to planet revealed it to be a dead world covered by rock and dust. Still, thanks to its extensive in-situ study with orbital probes but also landers and rovers, we now know that large amounts of liquid water did exist on Mars' surface about 3.8 billion years ago. Some liquid water probably still exists in the Martian sub-surface, maybe providing conditions well-suited for microbes to survive. The exploration of the Martian subsoil is thus the focus of several space missions to come. But whatever those missions will discover, Mars will not gain the status of habitable world. This is also the case for the other planets of the solar system. Mercury has revealed to be a tiny atmosphere-less planet baked by the Sun. Venus is a twin of the Earth in terms of mass and radius, but its smaller distance to the Sun resulted in a very dense atmosphere composed mostly of carbon dioxide that traps heat in a runaway version of the greenhouse effect that warms Earth's surface. As a result, temperatures and pressures on the surface of Venus are extreme (mean values of 460 degrees Celsius and 90 atmospheres), which, when combined with the absence of water and nice rains of sulfuric acid, make the planet totally inhospitable to any form of life. On their side, Jupiter, Saturn, Uranus, and Neptune are cold gigantic balls of gas without any solid surface. These giant planets have many moons, and some of them harbor probably large amounts of liquid water (e.g. Europa, Ganymede, or Enceladus), but under a very thick crust of ice.

For what we know, some microbes could hide in Mars sub-surface or in the subglacial oceans of Europa and a few other moons, yet Earth is definitely the only world of the solar system harboring a complex surface biosphere and hosting multicellular organisms intelligent and curious enough to wonder about their uniqueness in the large cosmos. To find other creatures of a similar nature, we have no choice but to look much further way, to the stars.

\section{Dreams of extrasolar civilizations}


In dark and clear conditions, one can observe a hazy band of white light arching across the night sky, the Milky Way. Thanks to his telescope, Galileo Galilei could resolve it and discover that it is composed of a huge number of stars. Later observations enable astronomers to show that the Milky way is a flattened rotating disk composed of hundreds of billions of stars including the Sun - held together by gravitational forces, similar to the solar system, but on a much larger scale. It was first thought that the Milky Way, called also the Galaxy, contained all the stars of the Universe. Still, more observations gathered in the first part of the twentieth centuries showed that some nebulae thought to belong to the Milky Way were in fact independent cluster of stars much further away, i.e. other galaxies. With the advent of more and more powerful telescopes, twentieth century astronomy revealed that the Universe contains hundreds of billions of galaxies, each composed of hundred million to hundred trillion stars. This led to the astonishing conclusion that "the total number of stars in the Universe is greater than all the grains of sand on all the beaches of the planet Earth." (Carl Sagan, 1980).

The revelation that Mars was a dead inhospitable world combined to the understanding of the structure of the Milky Way made the imagination of science-fiction (Sci-Fi) writers turn towards the stars. Stories of advanced aliens coming from beyond our solar system to visit or invade- us, or of humans of the future exploring exoplanets inhabited by exotic forms of life flourished in the first part of the twentieth century, notably in "pulps". These later were inexpensive fiction magazines very popular at that time, especially in the USA. A pioneer in this respect was H. P. Lovecraft (1890 - 1937), an American writer whose pulp fictions remained mostly unknown during his lifetime, but who is now consider as one of the most influential authors of horror and Sci-Fi of the twentieth century. Lovecraft's universe is full of horrors that humans should better not try to comprehend. Notably, many of his novels involves very ancient malevolent creatures -or deities- arrived from outer space eons ago, and who now hide in the darkness to plot against humanity.

After Lovecraft came two legendary Sci-Fi authors with two totally different views of life in our galaxy, and whose first works were also published in pulps. The first of them was Isaac Asimov (1920 - 1992), a former Professor of biochemistry who imagined in his Foundation and Robot series the future of humanity, with our descendants spreading to the stars to form an all mighty (but still fragile) galactic empire. In Asimov's novels, life is relatively common in the Milky Way, but it remains mostly composed of microbes or simple - and weakmulticellular organisms, Earth being a galactic exception. The second author was Arthur $C$. Clarke (1917 - 2008), a writer and inventor graduated in physics and mathematics with a strong passion for space. Contrary to Asimov, Clarke imagined a galaxy that is not only full of life, but that also hosts civilizations much older and more technologically advanced than ours.

Contact with very advanced sentient aliens became a widespread science-fiction theme in the second part of the twentieth century, notably with movies like 2001: A Space Odyssey (1968), Close Encounters of the Third Kind (1977), or ET (1982). Surprisingly enough, it also became a matter of national security and public concern soon after the end of World War II. In 1946, thousands of sightings of mysterious "ghost rockets" were reported in Sweden, in Greece, and in other countries. In summer 1947 came a rash of "flying saucers" all over the USA, leading to a kind of hysteria that went on for several years. In reaction, the US government undertook several studies of these sightings. The first of them, Project Sign, reached the astonishing conclusion that a fraction of the sightings were due to real artificial flying objects of 
extraterrestrial origins (!). Unsupported by any strong evidence, this conclusion was dismissed by the Pentagon, but official investigations went on until 1969 (Project Grudge, then Project Blue Book). Their termination was preceded by a scientific study performed by the University of Colorado, under the lead of the physicist Edward Condon (1902-1974). It concluded that most sightings of "Unidentified Flying Objects" (UFOs) were due to misinterpretation of conventional or natural phenomena combined to a mild form of mass hysteria, and that there was not a single piece of evidence of the visitation of our planet by aliens. This conclusion has remained valid until now, despite that UFO sightings have went on being reported all over the planet.

\section{But, seriously, where are the aliens?}

In 1950, the flurry of reported UFO sightings inspired the famous physicist Enrico Fermi (1901 -1954), who did not believe at all in their extraterrestrial origins, to wonder why our Earth is not really visited by aliens. This 'Fermi's Paradox' could seem ridiculous at first, but it is in fact a totally relevant question when considering the following points:

- There are (or have been) dozens of billions of potentially habitable exoplanets in our galaxy, a fact that was only suspected at the time of Fermi, but which is now firmly established, as discussed below.

- All the elements required by life on Earth are ubiquitous in the Universe.

- Life seems to have appeared on Earth between 3.8 to 3.5 billion years ago, i.e. soon after the planet was cold enough to harbor large stable reservoirs of liquid water on its surface.

- Globally, life on Earth has evolved from simple unicellular organisms to very complex multicellular creatures composed of billions of specialized cells. This evolution has been ruled by natural selection under varying environmental and ecological conditions. This Darwinian evolution mechanism corresponds to a gradual exploration of 'solutions' adopted by live forms to resist enough selection pressures to reproduce, and thus to survive. This mechanism is highly convergent, i.e. many analogous 'solutions' have been selected multiple times during the course of the history of life, suggesting that the number of solutions that 'work' in Earthlike conditions is limited. This conclusion also applies to advanced cognitive traits, an evolutionary advantage that has been selected independently by many animal species during the last 500 million years. Given humans' evolutionary success, it is thus tempting to assume that a cognition level large enough to master advanced technology, like the one of humans, has a significant probability to be selected by natural selection at some point or another in any stable Earth-like biosphere.

- Given the vast distance between the stars, interstellar travel would require spaceships able to reach at least a few percent of the speed of light, to make possible to a space probe to reach the nearest star within a few decades. This implies an improvement of a factor ten thousand relative to the speed of our fastest spacecrafts. The required technological leap is thus gigantic, but, given our past and current levels of technological developments, it seems reasonable to assume that this leap could be performed within a few centuries, a negligible duration compared to the age of our species (a few hundred thousand years).

- Even with spaceships travelling at 'only' one percent of the speed of light, the whole Milky Way could be completely explored by a civilization within a few dozens of million years, a duration three orders of magnitudes smaller than its age (13 billion years). 
Based on these considerations, Fermi's question is totally valid. Its relevance increases even further when considering the concept of 'Van Neumann probes', i.e. robotic interstellar probes able to use the material found in their target planetary system to reproduce themselves, send several clones of themselves to other nearby systems, and so on. With such a strategy, the exploration of the galaxy would reach an exponential rate similar to the colonization of a Petri box by bacteria, and could be accomplished within less than a million years.

Many speculative explanations to Fermi's paradox have been proposed. As Fermi was working on the US military nuclear program, the legend says that his favored hypothesis was that civilizations tend to self-destruct once they have mastered the power of the atom, and that their lifetime is always too short to enable them to spread to the stars. Since the end of the Cold War, the most commonly cited hypothesis is the 'Great Filter': the evolution of life towards a technologically advanced species implies at least one event of extremely low probability, even when integrated over billions of years. In such a scenario, life could be widespread in the galaxy, and still the probability for another species to have reached a level of cognition similar to ours is nearly zero. In other words, we are alone, or too isolated to ever establish a contact. A second hypothesis, even darker and still deemed likely by some scientists, implies that one of the first technologically advanced civilizations of the Milky Way could have not only explored the whole galaxy with its self-replicative probes billion years ago, but it could also have programmed them to destroy all other civilizations about to master interstellar travel, to avoid any potential competition with another intelligence. In such a scenario, we have always been spied by stealth alien probes ... and we are doomed by our constant technological progress and our dreams of travel to the stars.

In front of the two solutions cited above, we can only agree with Arthur C. Clarke when he wrote: "Two possibilities exist: either we are alone in the Universe or we are not. Both are equally terrifying.". Still, there is a third, less dark, solution that is also often cited. It implies the impossibility of interstellar travel, for technological or even economic reasons. This solution prevents, alas (or not), any direct contact between two civilizations, but not them to communicate between each other across the interstellar void, e.g. by using strong radio emissions or laser pulses. This is the speculation that motivated the project SETI, the Search for Extraterrestrial Intelligence. In their 1959 seminal paper, Giuseppe Cocconi and Phil Morison advocated a search for artificial radio signals emitted by a nearby civilization trying to contact us. It was soon followed by the suggestion to search for pulsed optical laser signals (Schwartz \& Townes1961). These suggestions have led to more than a hundred observational projects (see Tarter 2001 for a review), the first of them being conducted by Frank Drake in 1960. Several SETI projects are still ongoing today. So far, heavens have remained silent.

\section{How are planets formed?}

The existence of planets around other stars than the Sun remained pure speculation until the end of last century. While the hypothesis of abundant planetary systems was deemed likely by most astronomers since the time of Newton, it went into serious attack in the early twentieth century, with the advent of new models for the formation of the solar system that implied an extremely unlikely event, similar to the 'Great Filter' hypothesis used by some to explain Fermi's paradox. This assumed event is the close encounter -or even the collision- of the young Sun with another star, causing some material of one of the two stars to be ejected 
under the form of filaments trapped around the Sun by its gravity. These filaments would then have condensed into the planets of the solar system. Because stars are so scarcely scattered in space, such a stellar collision should be an extremely rare event, making our planetary system a cosmic oddity, and life elsewhere in our galaxy extremely unlikely.

The situation changed abruptly from 1935, first with theoretical attacks to the encounter/collision hypothesis that his supporters could not parry, then with the announced detections of exoplanets (Strand 1943, Reuyl \& Holmberg 1943) around some nearby stars, and finally with the advent of a new generation of models for the formation of our solar system (von Weiszäcker 1943, Struve 1950, Kuiper 1951). While the exoplanets detections of 1943 were finally discarded, as several previous and subsequent ones, the theoretical understanding of the formation of our planetary system improved gradually, to lead to the current paradigm model of "core-accretion" (Safranov 1969, Goldreich \& Ward 1973). In this model, stars form from the gravitational collapse of dust and gas (mostly hydrogen and helium) within an interstellar nebula named 'molecular cloud'. While contracting, the falling material forms a flattened disk surrounding a very hot blob that eventually reaches in its core temperatures and pressures large enough to sustain the nuclear fusion of hydrogen, becoming then a star. Within the surrounding disk, dust particles coagulate to form larger and larger bodies by accretion: pebbles, boulders, $\mathrm{km}$-sized objects (called planetesimals), Moon- to Mars-sized bodies (called protoplanets), and finally bona fide planets. The process is complex, as it implies several stages, the gravitational interactions between all these bodies and the surrounding gaseous component of the disk, the gravitational and electromagnetic influences of the central proto-star, inward/outward migrations, collisions, etc. The gas part of the disk is gradually eroded by the high-energy irradiation of the proto-star, and has totally disappeared within a few million years. If a planet reaches a mass of about a few Earth masses while the gas is still around, it starts accreting the surrounding gas in a runaway mode, turning quickly from a solid planet into a gas giant. Such a transformation is more likely to occur in the outer parts of the disk, where the density of solid material is much larger. Indeed, the closer to the young star, the larger the temperatures, and the most refractory a material has to be to remain solid.

If valid, the core-accretion model implied that planets are the natural by-products of the formation of stars. This inference got its first pieces of confirmation in the eighties, with the first direct detection of disks of cold dust and gas around young stars. As more results were obtained, it became clear that such circumstellar disks are ubiquitous around nascent stars. But how frequent was the formation of planets within such disks was still matter of debate, as the first confirmed detection of an exoplanet remained elusive.

\section{The exoplanets revolution}

After more than a century of failed attempts and false detections, first exoplanets were finally discovered in 1992, but around a star very different to our Sun (Wolszczan \& Frail 1992). Indeed, these planets of a few Earth masses orbit a pulsar, the tiny but extremely hot remnant of the supernova explosion of a dying massive star. It is not yet clear if these planets existed before the explosive agony of their host, or were formed afterwards from the expulsed material. Because of the exotic nature of their host, the discovery of these planets got mostly unnoticed outside the astronomers' community. But it was not the case at all for the Jupiter- 
like planet discovered in 1995 around a nearby Sun-like star (Mayor \& Queloz 1995). After centuries of speculation, this seminal discovery confirmed finally that planets were not exclusive to the solar system, opening a new era of modern astronomy. But like in 1992, the discovery came as a surprise, this time not because of the nature of the star, but because of the super-short orbit of the planet. Indeed, it takes only four days to make a full orbit vs eleven years for Jupiter around the Sun, and its distance to its star is only one percent of the JupiterSun distance. Because of this proximity, it must be extremely irradiated by its host star, which made it become the prototype of a new class of planets called "hot Jupiters". There is not enough material so close to the star in any protoplanetary disk to form such a massive planet, thus it must have formed much further out and then migrated inwards, something that was not anticipated by most planet formation models designed to reproduce the structure of our solar system.

In the next five years, a few more giant exoplanets were discovered around nearby stars, most of them in short-period orbits. Most of these planets were discovered by the co-called radial velocity method (Lovis 2010) which relies on the periodic motion of a star orbited by a planet around their common center of mass. It is thus an indirect method: the light of the planet is not measured, but only its gravitational influence on its host star. In 2000, a hot Jupiter previously discovered by this technique was revealed to transit, i.e. to pass in front of its host star once per orbit as seen from the Earth's perspective (Charbonneau et al. 2000, Henry et al. 2000). This transit corresponds to a partial eclipse of the star, similar to the transits of Venus or Mercury in front of the Sun. Exoplanets are light-years away, making impossible to image their crossing in front of the disk of their host star, unlike for Venus and Mercury. Still, the transit can be indirectly detected by measuring very precisely the apparent brightness of the star. When the planet passes in front of the star, it is partially occulted and appears dimmer. This purely geometric phenomenon makes possible not only to detect exoplanets, but also to measure their sizes. Indeed, the bigger the planet, the largest the occulted fraction of the stellar disk, and the largest its dimming. By combining the transit and radial velocity methods, one can measure also the absolute mass of an exoplanet, and when combined with its size, its density, a strong indicator of its bulk composition (Winn 2010). Applying these techniques to the first transiting planet confirmed that it was well a low-density gas giant similar to Jupiter.

During the last two decades, the field of exoplanet detection has evolved dramatically, and more than four thousand exoplanets have been discovered at an ever-increasing rate by more and more ambitious new projects from the ground and from space. Most of these exoplanets were detected by the transit method, a significant fraction by the Kepler space mission launched by NASA in 2009. Thanks to this huge number of known exoplanets, first important conclusions could be reached about the frequency and diversity of planetary systems. The most important of them is that Giordano Bruno was right: most stars of our Milky Way, and by extension of the whole Universe, are well circled by their own cortege of planets. Nevertheless, planetary systems are much more diverse than previously anticipated. About thirty to fifty percent of Sun-like stars are surrounded by a compact system of several planets of a few Earth masses in orbits shorter than the one of Mercury, the innermost planet of our own system. Up to a few percent have one Jupiter-like planet in a very elongated orbit, unlike the planets of our solar system that all have a nearly circular orbit. Some planets orbit around two stars. Some have no host star at all, they are freely floating in the interstellar void, 
probably after having been ejected from their initial planetary system. Systems similar to ours are estimated to be found around less than five percent of the Sun-like stars of our galaxy. Planetary formation is well the natural by-product of stellar formation, but it is a very complex and chaotic mechanism with a very wide diversity of outcomes (Winn \& Fabrycky 2014).

\section{Exoplanets and life}

Among the thousands of known exoplanets, a few dozen are potentially habitable, i.e. they could be rocky planets harboring large and stable reservoirs of liquid water on their surfaces ${ }^{1}$. The main conditions for a planet to be habitable are its composition and its distance to its host star. If the planet is a gas giant, it lacks a solid surface for liquid water to exist on. A habitable planet has thus to be rocky, i.e. to have a composition dominated by metals, rocks, and possibly ices (volatile elements like water, methane, and ammonia). Too close to the host star, the planet' surface liquid water will totally evaporate, while too far it will freeze. There is thus a limited range of distances to the star for which water could exist in liquid form on a rocky planet. It is called the habitable zone of the star, a concept that already appears in Newton's Principia, and presented in its modern form in 1953 by Hubertus Strughold. Its inner and outer limits can only be approximated, as they depend critically on the properties of the planet, and notably of its atmosphere. For instance, if the atmosphere is very dense, its greenhouse effect could be very strong, and the planet could harbor surface temperatures well above the evaporation temperature of water even if it is only mildly irradiated by its host star. Oppositely, if the atmosphere is too scarce, the pressures and temperatures at the surface could be too low to enable water to exist in liquid form. For these reasons, the computation of the limits of the habitable zone assumes generally an Earth-like planet with an Earth-like atmosphere. Applied to the solar system, such computations predict inner and outer limits of 95\% and 170\% of Earth's average orbital distance (Kopparapu et al. 2013). Both Earth's and Mars' orbits lie within these limits. This shows an important point: the actual habitability of a planet depends on much more parameters than its rocky nature and its distance to its host star. Because of a mass of only $10 \%$ of Earth's, Mars' interior cooled down much quicker than the one of our planet, and at some point its metallic core got cold enough to solidify. The shielding effect of the global magnetic field of the planet was lost then, and the atmosphere of Mars started to be strongly eroded by the Sun's high-energy photons and winds of charged particles. In the process, surface liquid water evaporated completely and was lost to space ${ }^{2}$, leaving eventually a desert world surrounded by a very thin atmosphere.

Many other ingredients should come into play to make a rocky planet habitable or not, e.g. the type of its host star and its magnetic activity, the rotation rate of the planet, the orientation of its spin axis, the presence of a moon around it, the rate and magnitude of impacts on its surface, and even the distance of the system to the center of the galaxy. For the few dozen of known potentially habitable exoplanets, all we know is that they orbit within the habitable zone of their stars, and that their measured masses and/or radii are consistent with a rocky composition. That is way too little to conclude that these planets are actually habitable. As sending probes to these planets to study them in-situ is impossible with our

\footnotetext{
${ }^{1}$ The term "habitable" is particularly ill chosen as it refers in common talk to a place that is suitable to live in, while here it refers only to the existence of surface water in liquid form.

${ }^{2}$ Actually, the evaporated water molecules were "photolyzed" by the high-energy light of the Sun, the freed atoms of hydrogen got lost to space, while most of the remaining oxygen atoms reacted with the surface ironrich rocks, giving them eventually their red color.
} 
current technology, the only way to assess these planets' actual habitability is to measure their atmospheric properties and compositions from afar. Indeed, there is an intimate link between a planet's surface conditions and atmospheric compositions, as discussed above. By measuring accurately enough the extent, density, and composition of the atmosphere, and combining this information with accurate measurements of the planet's mass, size, and orbital parameters, and of its host star's properties, one should be able to infer if liquid water is likely to exist on its surface or not.

In fact, measuring the planet's atmospheric composition could reveal the existence of life itself. Indeed, living creatures and their environment form a complex integrated system, called biosphere. The Earth's atmosphere is a key component of its biosphere, and its composition has been strongly influenced by the emergence and evolution of life during the planet's history. For instance, molecular oxygen represents about twenty percent of Earth's atmospheric composition, and it is mostly sourced from photosynthesis, the process used by planets and other organisms to convert the energy of the Sun's light into usable chemical energy. An alien astronomer located light-years away could thus infer that our planet hosts photosynthetic organisms just by measuring the high oxygen content of its atmosphere. For that reason, molecular oxygen is what is called a potential biosignature, i.e. a substance whose origin could be related to a biological agent. Several other simple molecules are also potential chemical biosignatures, e.g. ozone, methane, or nitrous oxide.

Studying remotely the atmospheric properties of a terrestrial exoplanet could thus in principle enable us to constrain its surface conditions, and event to detect chemical traces of life. But how to do that? The key technique to apply here is called spectroscopy, the separation of the light of an object by its wavelengths (i.e. its colors) and the recording of the resulting data. These latter consist in the distribution of the target's light as a function of the wavelengths, what is called a spectrum. The light emitted by a terrestrial planet originates from the reflection of a fraction of the light received from its host star, and from the intrinsic emission of its surface and hottest atmospheric layers. In both cases, the emitted light will be altered by the planet's atmosphere, keeping key imprints of its composition and structure in its wavelength distribution. For instance, measuring the infrared spectrum of Mars, Venus, and Earth, one can notice patterns that can be related to carbon dioxide for the three planets, but also to water and ozone for Earth, ozone being the by-product of the photolysis of highaltitude oxygen molecules by the UV photons from the Sun.

With spectroscopic data of sufficient quality, the thorough atmospheric characterization of a planet is thus possible. The path to detect life around other stars is thus a well-defined twosteps process: first, detecting potentially habitable exoplanets, and then studying their emitted light by spectroscopy to measure their atmospheric compositions, decipher their surface conditions and, possibly, detect biosignatures. This second step is, unfortunately, out of reach with current technology. Indeed, it requires an extremely challenging task: not only to measure the light of a very faint planet located light-years away, but also to separate it from the light of a much brighter object which is separated of the planet by a tiny angular distance: its host star. The Earth is hundreds million times fainter than the Sun, while its maximal angular distance to the Sun as seen from the nearest star would be smaller than a second of arc, i.e. 1/3600 of a degree! Attempting to detect the light of the Earth from the nearest star is thus similar to trying to resolve with googles the light of a firefly landed on a powerful lighthouse kilometer away. Such an achievement requires optical techniques able to strongly attenuate the light of stars while enabling to detect very faint sources in their vicinity. 
Those high-resolution high-contrast imaging techniques are called adaptive optics, coronography, nulling interferometry, etc (Traub. \& Oppenheimer 2010). They are quickly developing, and have already made possible the detection of several dozens of massive gas giant exoplanets in wide orbit around their host stars (e.g. Marois et al. 2008, see Fig. 1). Detecting and studying a real Earth-twin with these techniques will surely be possible at some point, but probably not before a few decades and significant technological developments.

Nevertheless, a promising shortcut exists...

\section{Transits, ultracool dwarf stars, and TRAPPIST-1}

If a planet transits its host star, we have seen that it can be detected indirectly by the resulting drop of apparent brightness of the star, and that its size can be determined from the amplitude of the drop. But there is more. During the transit, a small fraction of the light emitted by the star towards Earth will pass through the planet's atmosphere, and will be filtered by it. With spectroscopic data of sufficient quality, the resulting alteration of the light of the system could be in theory detected and make possible to constrain the atmospheric composition of the planet. Furthermore, a planet that transits has its orbital plane nearly parallel to the line connecting Earth to its host star. This means that every half-orbit after its transit, it should pass behind the star as seen from Earth, and be totally occulted by it. The corresponding drop of apparent brightness could be measured in different wavelengths, making possible to reconstruct the emission spectrum of the planet without even resolving it from its host star. In addition, by measuring how the global light of the system is modulated during a full rotation of the planet, extra-information on the planet's atmosphere and weather can also be obtained. Combining these three methods (Winn 2010), called transit, occultation, and phase curve spectroscopy, and assuming sufficient precisions, it should be theoretically possible to decipher the atmospheric and surface properties of a potentially habitable exoplanet. Such atmospheric studies have already been performed with ground- and spacebased telescopes for a few dozen hot Jupiters, i.e. short-period giant planets (e.g. Cowan et al. 2015).

The challenge is now to export these methods to transiting potentially habitable rocky planets. Unfortunately, this cannot be done for real Earth-twins, i.e. Earth-like planets in Earth-like orbits around Sun-like stars. The reason is the huge contrast in size and temperature between an Earth-twin and a Sun-like star. For instance, with the transit spectroscopy method, the fraction of the light of a Sun-sized star passing through the thin atmospheric limb of an Earthlike planet is ridiculously small (less than one part-per-million), and the resulting tiny spectroscopic signatures from the planet's atmosphere beyond the reach of our instrumental technology. Still, there is hope. Indeed, the Sun is pretty big and hot compared to most stars of the Milky Way. The smallest and coolest stars, called ultracool dwarf stars, represent about fifteen percent of the galactic population. Their size is about ten times smaller than the Sun, similar to Jupiter's, and their surface temperature is more than twice smaller than the Sun's. For such a cold and tiny star, the atmospheric characterization of a temperate Earth-sized rocky planet should be within reach of the next generation of giant telescopes, notably of the upcoming NASA James Webb Space Telescope (JWST, Fig. 2) to be launched in 2021 (e.g. Morley et al. 2017).

While most transit searches have focused on Sun-like stars, it is thus highly desirable to explore also the nearest ultracool dwarf stars for transits, so to detect potentially habitable 
worlds that are well-suited for detailed atmospheric studies and biosignature searches with JWST and other future instruments. This is the goal of our project SPECULOOS (Gillon 2018, Delrez et al. 2018). It is based on a network of robotic ground-based telescopes that will perform a transit search on the one thousand nearest ultracool dwarf stars. The core facility of the project, the SPECULOOS Southern Observatory (Delrez et al. 2018), has just started its operations last January at the European Paranal Observatory in the Atacama Desert of Chile (Fig. 3). Still, the project started in fact in 2011 as a prototype using TRAPPIST, a small robotic telescope located in Chile, to monitor the brightness of a limited sample of fifty nearby ultracool dwarfs (Gillon et al. 2013). This prototype aimed only to assess the feasibility of SPECULOOS, notably in terms of precision and variability of these small stars. It did much better than that. Indeed, in 2015, it detected three transiting Earth-sized planets in orbit around a Jupiter-sized star forty light-years away in the Aquarius constellation (Gillon et al. 2016). In 2016, our intensive follow-up monitoring of the star -renamed TRAPPIST-1- revealed that it is in fact orbited by seven Earth-sized planets, all of them close or within the habitable zone of the star (Gillon et al. 2017). This amazing planetary system is super compact, the distance of the planets to the star ranging from one to six percent the Sun-Earth distance, and their orbital periods being comprised between 1.5 and 21 days (Fig. 4). The minimal distance between two adjacent planets is only a few times the Earth-Moon distance. Still, despite this extreme proximity of the planets, the system is probably very stable, thanks to it peculiar dynamical architecture. Indeed, the orbital periods of the planets are commensurate, i.e. their values are related by ratios of integer numbers (Luger et al. 2017). Thanks to this architecture, a push of one planet on another is always nearly compensated by an opposite push from another planet. Hence, the planets interact strongly with each other, but in a way that the sum of their interactions average out. In our solar system, such a 'resonant' structure can be found for some moons of giant planets, notably for lo, Europa, and Ganymede, the three inner main moons of Jupiter. And just like these moons, or the Moon itself, the tidal influence of their much more massive host should have trapped them in a synchronous rotation state, i.e. their rotation period should equal their orbital period, making them show always the same hemisphere to their star.

Thanks to our intensive follow-up campaign, we could measure very precisely the masses and radii of the seven planets (Grimm et al. 2017). The resulting densities suggest mostly rocky compositions, but with a much higher content in water than our planet. These compositions and the peculiar dynamical structure of the system make likely that the planets formed much further away from the star, in the outer water-rich part of the protoplanetary disk, and then migrated inwards by interaction with the gaseous part of the disk. Their irradiations by the star are moderate enough to make possible the existence of water in liquid phase on at least a fraction of their surfaces, especially for the three planets that orbit within the classical habitable zone of the star.

The study of the star itself revealed that it is probably older than the Sun. Still, like most verylow-mass stars, it has a strong magnetic activity which translates into an emission of highenergy photons (X-ray, UV) and charged particles (stellar wind) similar to the one of the much bigger Sun (Bourrier et al. 2017). As the planets are very close to the star, they are much more irradiated by X-ray, UV, and charge particles than the Earth. This high-energy bombardment could be detrimental to the planets' habitability. First, it could erode continuously their atmospheres, making them too scarce to make possible the existence of water in liquid form. Secondly, it could break chemical bonds and inhibit the making of the building blocks of life. 
Still, the hope for life remains. Indeed, the erosion of the atmosphere could be compensated by several outgassing processes, like the evaporation of surface volatile elements, volcanic activity, etc. Furthermore, atmospheric escape mechanisms are still poorly understood, and some of them could saturate under heavy irradiation. For instance, Venus is closer to the Sun than the Earth, and unshielded by a large magnetosphere similar to Earth's, and still the erosion of its atmosphere by the solar wind is negligible, thanks to motions of charged particles in its upper atmosphere that induce protective local magnetic fields (Zhang. et al. 2007). And even if the dayside surface is heavily irradiated by X-ray and UV photons, prebiotic chemistry could still happen under a protective layer of liquid water, and/or on the night side of the planet.

The next major step in the study of TRAPPIST-1 planets will start in 2021, with the launch of JWST. This $6 \mathrm{~m}$-aperture infrared space telescope, the largest telescope ever sent to space, should be able to probe the planets' atmospheric compositions, to constrain their surface properties and habitability, and even to detect molecules of possible biological origins (Morley et al. 2017). Other future potential space missions and giant ground-based observatories should also be able to refine our understanding of the planets. TRAPPIST-1 represents thus our first opportunity to search for traces of life beyond our solar system, but the bandwagon is now on the move, and many other opportunities are about to follow. SPECULOOS and other projects, like the TESS NASA space mission, should detect several other temperate terrestrial planets transiting nearby very small stars, extending the sample of potentially habitable worlds for which traces of life could be found in the near-future.

\section{TRAPPIST-1: from science to science-fiction}

We have seen that extrasolar worlds have been present in the science-fiction literature wellbefore the first actual detection of an exoplanet. Still, despite all their qualities, these fictional works failed to predict the diversity of planetary systems revealed during these last two decades by exoplanet search projects. It is thus no surprise that more and more SF novels of the "space opera" kind are inspired by actual exoplanet detection results. TRAPPIST-1 has been a perfect example of this tendency, from the very beginning. Indeed, the volume of the scientific journal Nature that contained our paper presenting the discovery of the seven planets system (Gillon et al. 2017) comprised also a short science-fiction story by the Swiss writer Laurence Shuner, taking place on the surface of one of the TRAPPIST-1 planets. Since then, TRAPPIST-1 has appeared in many science-fiction books, comics, and videogames. This intimate relationship between science and science-fiction should be no surprise. Indeed, both disciplines are basically two parallel methods to answer questions of the form: "what if...?", one basing on the confrontation between observations and theories, and the other combining our current scientific knowledge to the limitless power of imagination. Many scientists are thus fans of SF, finding there a great source of inspiration and creativity for their research. Oppositely, most SF artists have a strong passion for science, and their works are heavily inspired by the most recent scientific results or theories. In fact, it is not rare to see scientists dabbling into science-fiction, and even become successful science-fiction writers like, e.g. Isaac Asimov, Fred Hoyle, or Alastair Reynolds. It makes no doubt that this strong relationship will develop even further in the next decades, when the first detailed characterizations of potential extrasolar biospheres will be performed. 


\section{Life around other Suns: the near-future}

Among the many questions raised by human kind, only a few could be considered as truly fundamental. The possible existence of life elsewhere in the Universe is certainly one of them, and it is exhilarating to realize that we could be at the verge of answering it. Predicting the future is -at best- a hazardous enterprise, but it is undeniable that our first opportunities to find traces of life beyond our solar system will happen within one or two decades. If successfully launched and deployed, JWST should be able detect biosignatures in the atmospheres of some terrestrial planets transiting nearby ultracool dwarf stars, like TRAPPIST1 planets or those to be found by SPECULOOS. For slightly more massive and bigger stars called red dwarfs, it is possible that other projects, like the NASA TESS mission will soon discover transiting temperate "super-Earths", i.e. rocky planets slightly more massive and bigger than our planet, whose atmospheric exploration will also be within reach of JWST (Batalha et al. 2018). A couple of potentially habitable planets orbiting around nearby red dwarfs -like the one in orbit around Proxima Centauri, the nearest star- could also have their atmospheric composition explored in some detail by future giant telescopes currently under construction, even if they do not transit their host star (Hawker \& Parry 2019). If life is frequent around red and ultracool dwarf stars, we could thus detect it very soon.

Still, over-optimism should be avoided here. Indeed, one should keep in mind that the detailed properties of terrestrial exoplanets, especially in orbit around stars very different to the Sun, is an uncharted territory. Concluding with absolute certainty to the biological origins of some molecules detected in the atmosphere of a planet orbiting a low-mass star light-years away could well reveal to be a long process, requiring the comparative studies of a large sample of planets, complex modeling, and the systematic rejection of all possible alternative scenarios. Furthermore, it is well possible that life does not exist at all around red and ultracool dwarf stars, and is confined to more Sun-like hosts. If so, its detection will have to wait for the development of direct imaging technologies and the funding of ambitious space-based telescopes able to resolve the light of an Earth-twin to the one of its host star. Predicting when such a project will come true is a difficult exercise, but it is reasonable to assume that it will definitely happen before the end of the century, if not much before. Only a few planets will be within reach at first, and the sample will grow progressively, at a rate limited by the development of technologies and funding. And here too, the success of the enterprise will strongly depend on the frequency of life itself, and also on our still unknown capacity to discriminate biological and abiotic scenarios.

The next decades will thus be extremely exciting. First signs of extrasolar biology could thus be found pretty quickly (before 2035 ) if life is frequent around low-mass-stars, and later for more Sun-like stars. The full confirmation of their biological origins could take some more time yet. Even if life is NOT detected by 2100 , we should have then probed the atmospheres of enough extrasolar terrestrial worlds to draw some firm conclusions about life's prevalence in the Universe. In the meantime, the exploration of our own solar system will go on, and it could result in breakthrough results like the discovery of microbial organisms hiding under the surface of Mars, or complex ecosystems hidden beneath the icy crusts of some moons (e.g. Enceladus, Europa). SETI will pursue its search for artificial signals from outer space, and could reveal at some point that not only life exists elsewhere, but also that a more advanced civilization is trying to communicate with us. Inspired by these research projects, and inspiring them in the same time, the next generations of SF writers will go on telling us amazing stories of mysterious extraterrestrial creatures living under the light of other suns. 
Isaac Newton (1687), Philosophiæe Naturalis Principia Mathematica, edited by the Royal Society (London)

Nicolaus Copernicus (1543), De revolutionibus orbium coelestium, edited by J. Petreius (Nuremberg)

Bernard le Bouyer de Fontenelle (1686), Entretiens sur la pluralité des mondes, edited by C. Blageart (Paris)

Johannes Kepler (1634), Somnium, edited by L. Kepler (Frankfurt)

Christian Huyghens (1698), Cosmotheoros, edited by A. Moetjens (the Hague)

Herbert G. Wells (1898), The War of the Worlds, editer by W. Heinemann (London)

Carl Sagan (1980), Cosmos, Random House (USA)

Milan N. Církovic (2018), The Great Silence, Oxford (UK)

Steven J. Dick (1998), Life on other Worlds, Cambridge University Press (UK)

Giuseppe Cocconi \& Philip Morrison (1959), Searching for Interstellar Communications, Nature, 184, 844-846

R. N. Schwartz \& C. H. Townes (1961), Interstellar and Interplanetary Communication by Optical Maser, Nature, 190, 205-208

Jill Tarter (2001), The Search for Extraterrestrial Intelligence (SETI), Annual Review of Astronomy and Astrophysics, Vol. 39, p. 511-548

K. Strand (1943), 61 Cygni as a Triple System, Publications of the Astronomical Society of the Pacific, Vol. 55, No. 332, p. 29-32

Dirk Reuyl \& Erik Holmberg, On the Existence of a Third Component in the System 70 Ophiuchi, Astrophysical Journal, vol. 97, p. 41

C. F. von Weizsäcker (1943), Über die Entstehung des Planetensystems. Mit 2 Abbildungen, Zeit. Für Astrophys. 22, 319

Otto Struve (1950), Stellar Evolution, an exploration from the observatory, Princeton University Press (USA)

Gerard P. Kuiper (1951), On the Origin of the Solar System, Proceedings of the National Academy of Sciences of the United States of America, Volume 37, Issue 1, pp.1-14

Viktor S. Safronov (1972), Evolution of the protoplanetary cloud and formation of the Earth and Planets, NASA TT F-677 (originally published in Russian in 1969)

P. Goldreich \& W. R. Ward (1973), The formation of planetesimals, Astrophysical Journal, vol. 183, 1051-1061

A. Wolszczan A. \& D. A. Frail D. A. (1992), A planetary system around the millisecond pulsar PSR1257+12, Nature, vol. 355, 145

M. Mayor \& D. Queloz (1995), A Jupiter-mass companion to a Sun-like star, Nature, vol. 378, 355

C. Lovis (2010), Radial Velocity Techniques for Exoplanets, in Exoplanets, edited by Sara Seager, University of Arizona Press, p. 27 - 53 
D. Charbonneau et al. (2000), Detection of Planetary Transits Across a Sun-like Star, Astrophysical Journal, vol. 529, L45-L48

G. Henry et al. (2000), A Transiting "51 Peg-like" Planet, Astrophysical Journal, vol. 529, L41L44

J. Winn (2010), Exoplanets Transits and Occultations, in Exoplanets, edited by Sara Seager, University of Arizona Press, p. $55-77$

A. Cassan (2012), One or more bound planets per Milky Way star from microlensing observations, Nature, vol. 481, pp. 167-169

J. Winn \& D. Fabrycky (2014), The Occurrence and Architecture of Exoplanetary Systems, Annual Review of Astronomy and Astrophysics, vol. 53, pp. 409-447

Hubertus Strughold (1953), The Green and the Red Planet: A Physiological Study of the Possibility of Life on Mars, University of New Mexico Press (USA)

Wesley A. Traub \& Ben R. Oppenheimer, Direct Imaging of Exoplanets, in Exoplanets, edited by Sara Seager, University of Arizona Press, p. 111 - 156

C. Marois et al. (2008), Direct Imaging of multiple planets orbiting the star HR 8799, Science, vol. 322,1348

N. B. Cowan et al. (2015), Characterizing Transiting Planets Atmospheres Through 2015, Publications of the Astronomical Society of the Pacific, Vol. 127, Number 949

C. Morley et al. (2017), Observing the Atmospheres of Known Temperate Earth-sized Planets with JWST, Astrophysical Journal, vol. 850, Number 2

M. Gillon (2018), Searching for Red Worlds, Nature Astronomy, Vol. 2, p. 344

L. Delrez et al. (2018), SPECULOOS: a network of robotic telescopes to hunt for terrestrial planets around the nearest ultracool dwarfs, Proceedings of the SPIE, Volume 10700

M. Gillon et al. (2013), TRAPPIST/UCDTS : a prototype search for habitable planets transiting ultra-cool stars, in Hot Planets and Cool Stars, Garching, Germany, Edited by Roberto Saglia; EPJ Web of Conferences, Volume 47, id.03001

M. Gillon et al. (2016), Temperate Earth-sized planets transiting a nearby ultracool dwarf star, Nature, Vol. 533, Issue 7602, pp. 221-224

M. Gillon et al. (2017), Seven temperate terrestrial planets around the nearby ultracool dwarf star TRAPPIST-1, Nature, Vol. 542, Issue 7642, pp. 456-460

R. Luger et al. (2017), A seven-planet resonant chain in TRAPPIST-1, Nature Astronomy, Vol. 1, id. 0129

S. Grimm et al. (2018), The nature of the TRAPPIST-1 exoplanets, Astronomy and Astrophysics, Vol. 613, id. A68

V. Bourrier et al. (2017), Temporal Evolution of the High-energy Irradiation and Water Content of TRAPPIST-1 Exoplanets, the Astronomical Journal, Volume 154, Issue 3, article id. 121

T. L. Zhang et al. (2007), Little or no solar wind enters Venus' atmosphere at solar minimum, Nature, Vol. 450, Issue 7170, pp. 654-656

L. Suhner (2017), The terminator, Nature, Vol. 542, Issue 7642, pp. 512 
N. E. Batalha et al. (2018), Strategies for Constraining the Atmospheres of Temperate Terrestrial Planets with JWST, The Astrophysical Journal Letters, Volume 856, Issue 2, article id. L34

G. A. Hawker \& I. R. Parry (2019), High-resolution spectroscopy and high contrast imaging with the ELT: looking for $\mathrm{O}_{2}$ in Proxima $b$, Monthly Notices of the Royal Astronomical Society, Volume 484, Issue 4, p.4855-4864 


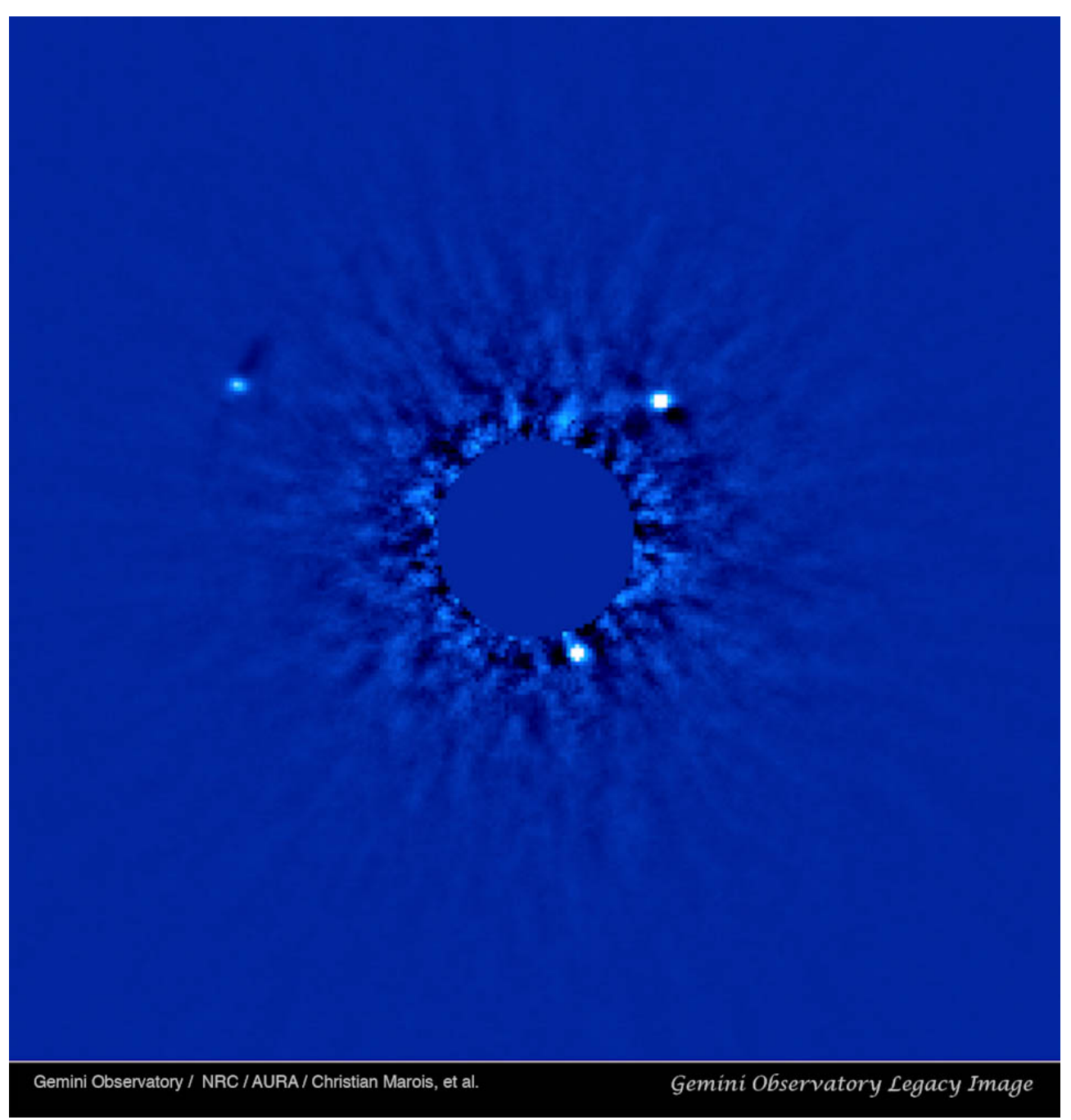

Fig. 1. Image of the HR8799 planetary system. The flux of the central star has been subtracted using a special observational and data analysis method to reveal the light of three of its planets (Marois et al. 2008). These directly imaged planets are more massive and are on much wider orbits than Jupiter. Furthermore, they are still young, hot, and bloated, which makes them much more luminous than Jupiter. Credit: Gemini Observatory/NRC/AURO/Christian Marois et al. 


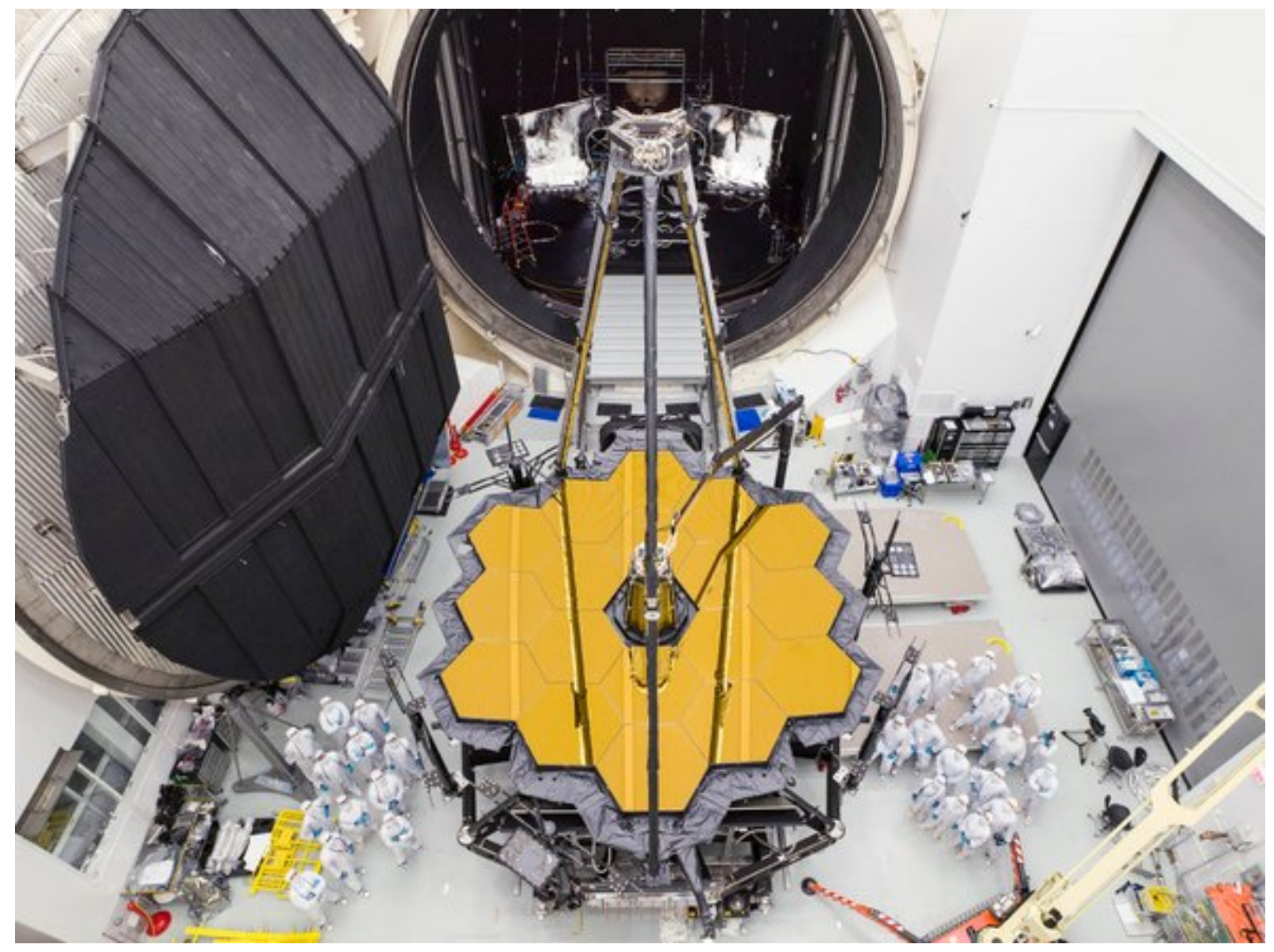

Fig. 2. The James Webb Space Telescope under test at NASA Johnson Space Center in December 2017. Credit: NASA/C. Gunn

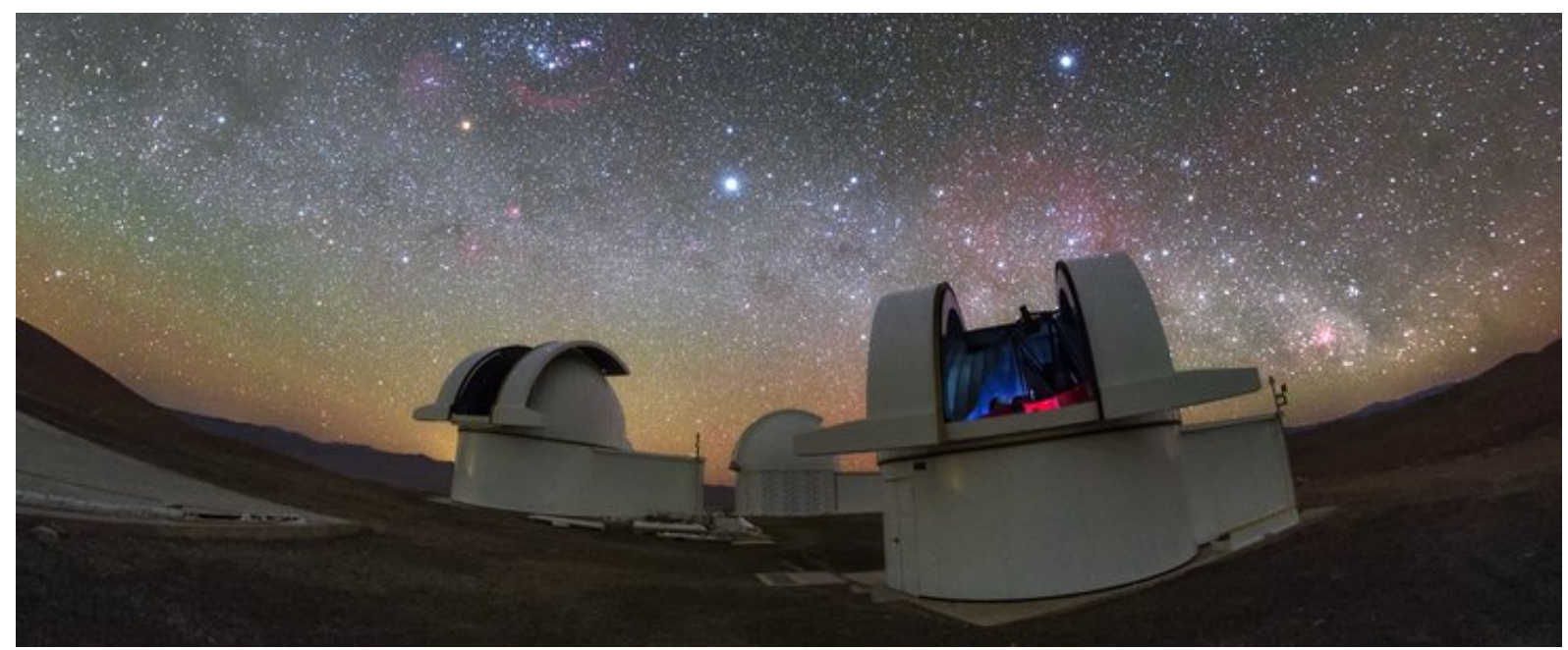

Fig. 3. The telescopes of the SPECULOOS Southern Observatory gazing into the night sky over the Atacama Desert (Chile). Credit: ESO/P. Horálek. 


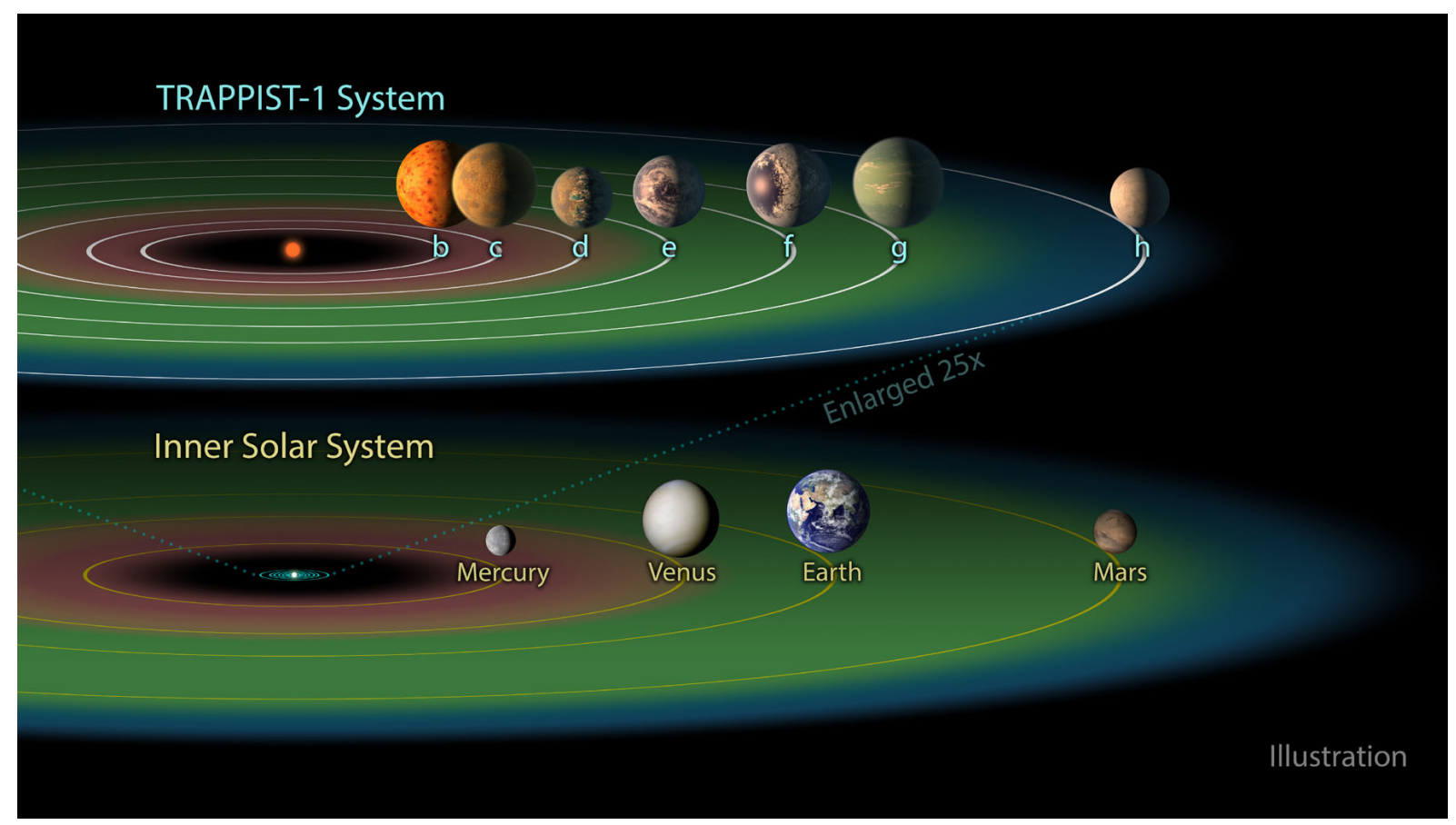

Fig. 4. Comparison of the TRAPPIST-1 system with the inner solar system. TRAPPIST-1 hosts seven Earth-sized planets within 7\% of the Earth-Sun distance. Three of those planets orbit within the habitable zone of the star. Credit: NASA/JPL-Caltech 